Supporting Information

\title{
Triboelectric nanogenerator from used surgical face mask and waste mylar materials aiding circular economy
}

\author{
Harris Varghese 1,2 and Achu Chandran 1,2 * \\ ${ }^{1}$ Materials Science and Technology Division, CSIR-National Institute for Interdisciplinary Science \\ and Technology (NIIST), Thiruvananthapuram-695019, India \\ ${ }^{2}$ Academy of Scientific and Innovative Research (AcSIR), Ghaziabad- 201002, India \\ Email: achuchandran@niist.res.in
}




\section{Surface morphology of UV-C treated PP material}

The surface morphology of UV-C treated non-woven PP fibres can be seen in the SEM image (Figure S1). From the SEM image it is clear that there is no damage to the fibre structure after disinfecting it with UV-C radiation and it is a non-destructive method.

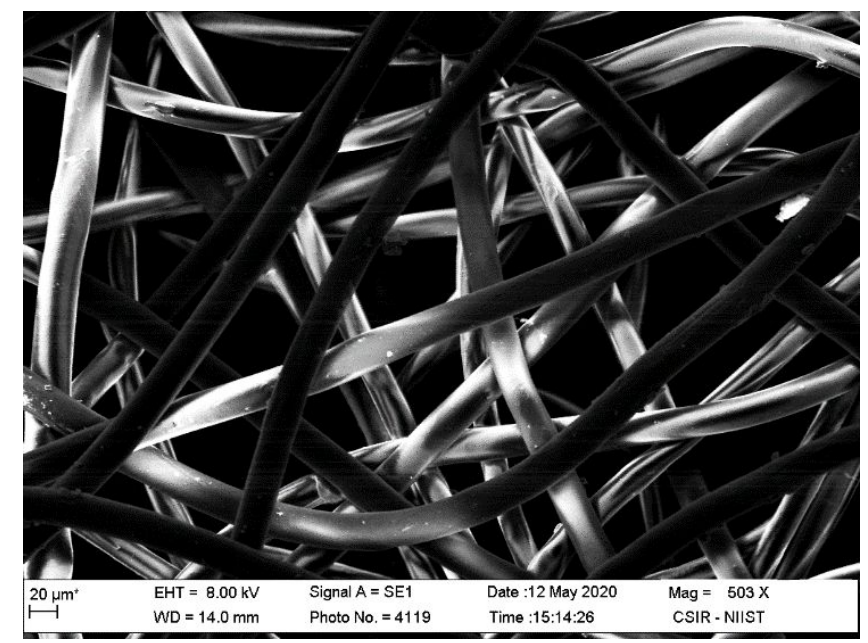

Figure S1: SEM image of PP surface after UV-C treatment

\section{Stability of Arc structure}

An arc shaped structure was also evaluated for the WM-TENG (inset of Figure S2). As it could be seen from the output given in Figure S2, the stability of the device was very poor. It showed reduction of about $100 \mathrm{~V}$ from time to time (after hundreds of cycles). The instability may be arisen due to the structure or the high separation gap of $2.5 \mathrm{~cm}$. Anyhow, this form factor was not considered for further optimization of the WM-TENG.

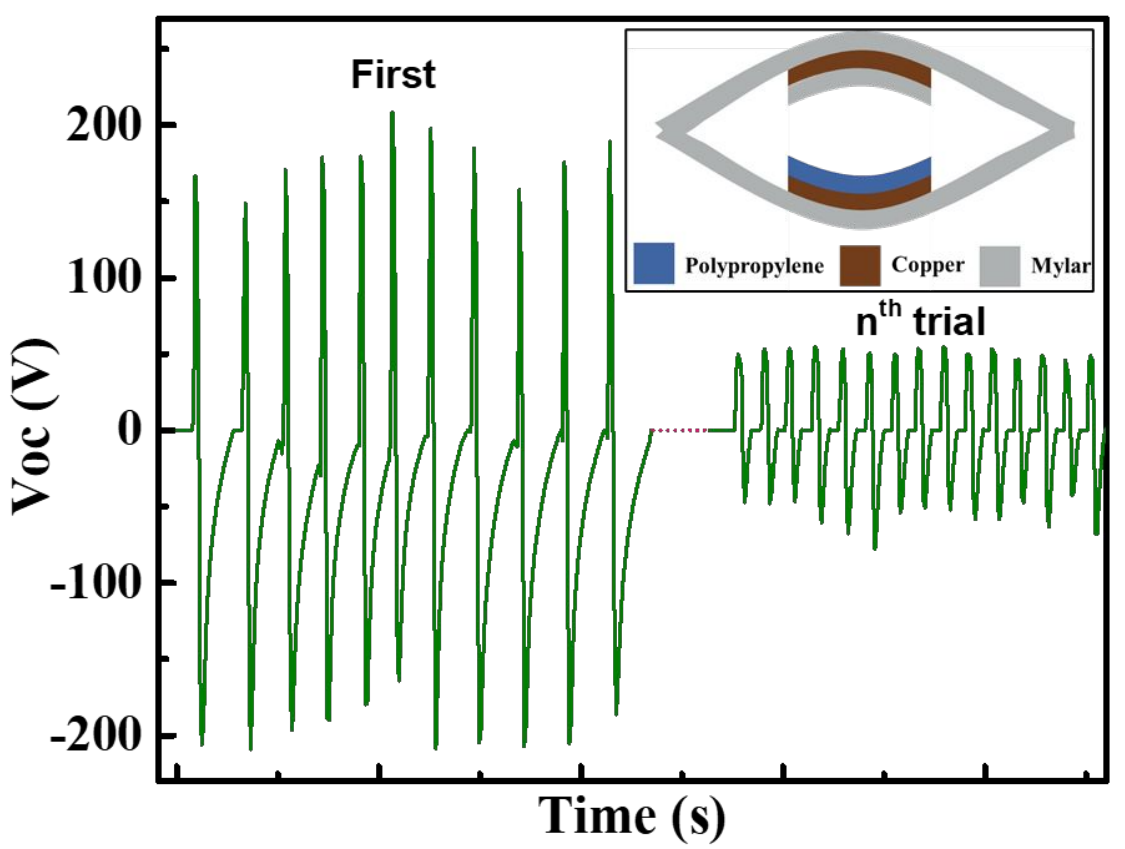

Figure S2: Open circuit voltage of WM-TENG in arc shaped structure (given in inset) showing its instability during hundreds of cycles of operation. 


\section{COMSOL simulation of WM-TENG}

The following simulation was done using the experimental value provided in the literature (reference 51), this experimental value is the maximum TECD value possible for the material pair at controlled conditions. It could be seen that the simulated potential is very much higher than that of the experimentally obtained one (Figure S3). So, it could be concluded that the TECD value obtained for the WM-TENG is lower than the maximum TECD possible for the pairs.

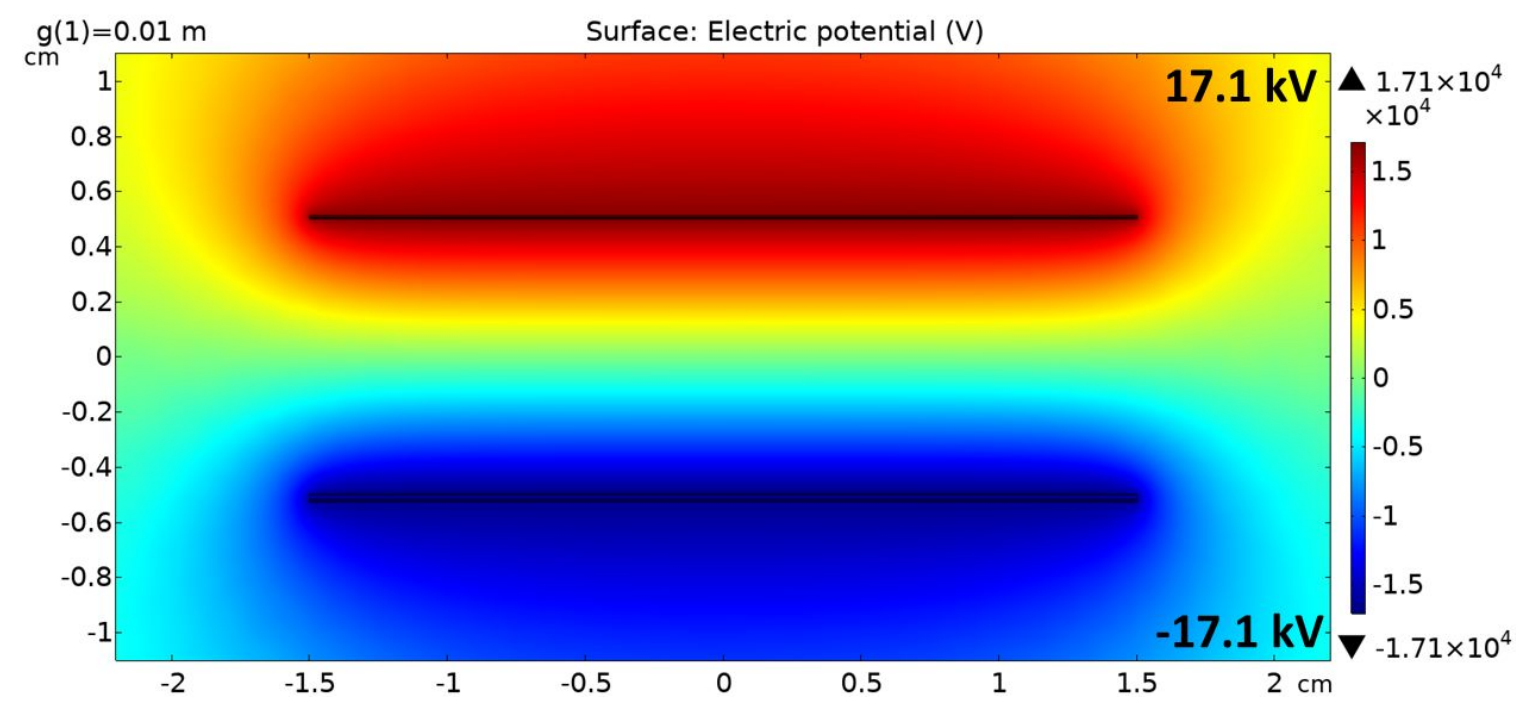

Figure S3: COMSOL simulation of potential of the WM-TENG using the TECD value from the literature.

\section{Measurement of force using a balance}

The force of mild finger tapping is measured roughly with the aid of an electronic balance as explained in the reference 56 on the main manuscript. Even though balances display mass of a body, it does not measure mass directly. Measurement of force or namely the weight force measurement is what the working principle of balances. Then the internal transducer converts it to the mass of the body according to the calibration factor. Here we do the opposite of this process by roughly taking the calibration factor as $1 / \mathrm{g}$ where $\mathrm{g}$ is the gravitational constant. The WM-TENG is mounted on top of an electronic balance and then it is pressed by exerting force with the finger. Then simultaneously both the mass readings and the output of the device is noted. Then the force required to get the proper output is calculated by multiplying the mass reading with $\mathrm{g}$. Thus, the force range is obtained as $0.4-1.2 \mathrm{~N}$. These contain errors due to the buoyancy and the calibration weight factor, however, these reading should suffice since finger tapping weight force changes from person to person. 


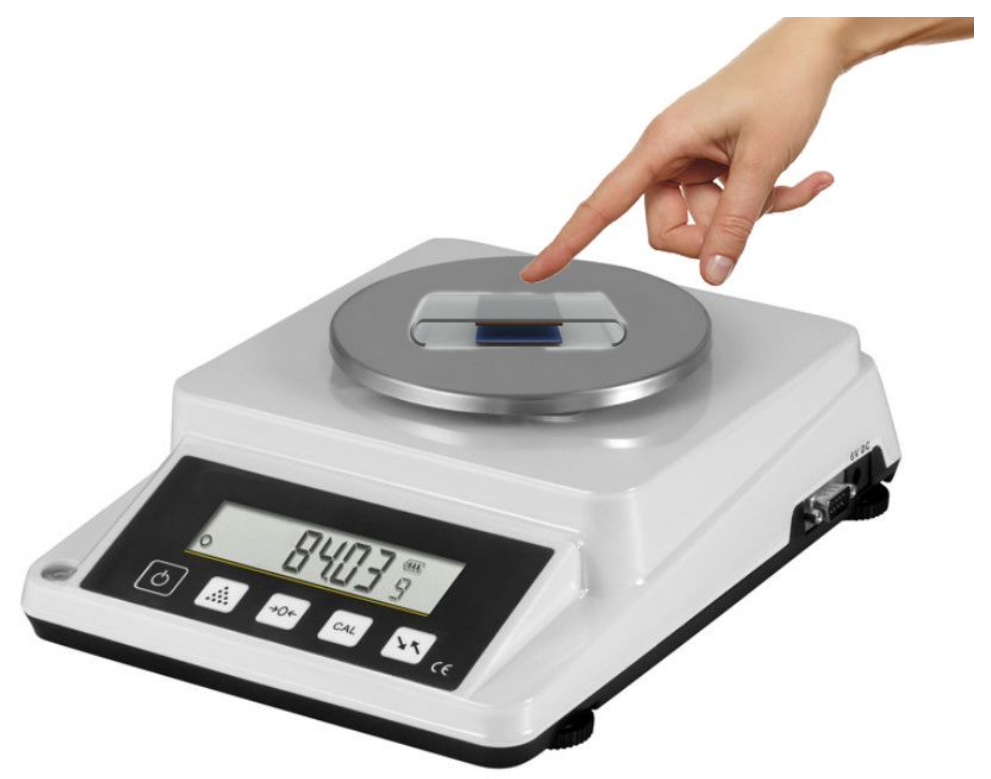

Figure S4: Measuring weight force using an electronic balance.

Movie 1: $\quad$ Powering a digital thermometer using WM-TENG to measure the room temperature.

Movie 2: $\quad$ Powering a calculator using WM-TENG to perform mathematical operations. 